\title{
Mean field theory calculations to model single crystal diffuse scattering
}

\author{
E.M. Schmidt, J.M. Bulled, A.L. Goodwin
}

Univerisy of Oxford, Inorganic Chemistry, South Parks Road, OX13QR

ella.schmidt@chem.ox.ac.uk

Correlated disorder in crystalline materials gives rise to single crystal diffuse scattering. While the average structure determination via Bragg data analysis is considered a standard procedure, disorder analysis is thought of as a lengthy and complicated process. We present a mean field approximation to model single crystal diffuse scattering in molecular materials from a simple pair-interaction Hamiltonian.

Mean filed theory is a self-consistent field theory, which is widely used in statistical physics to model high-dimensional random systems. It has proven a valuable tool in the analysis of magnetic diffuse scattering data [1]. Here, the formalism is applied to describe orientationally disordered molecular crystals,

We present a computational study based on the mean field model suggested by Naya [2] and proof its applicability to strongly correlated disorder, where the local building block geometry dictates allowed and prohibited local configurations. The system that will be analysed in detail is a two-dimensional analogue of $\mathrm{Hg}\left(\mathrm{NH}_{3}\right)_{2} \mathrm{Cl}_{2}$ as depicted in Figure 1 (a) [3]. The $\mathrm{Hg}$ atoms are disordered over the cubic face centres to form $\left[\mathrm{H}_{3} \mathrm{~N}-\mathrm{Hg}-\mathrm{NH}_{3}\right]^{2+}$ molecules. The local arrangement is strictly dictated by these building rules.

We compare the results of the diffuse scattering analysis using the mean field model as introduced by Naya [2] to the results of RMC modelling and $\triangle \mathrm{PDF}$ models based on a Warren-Cowley short range order parameter refinement (see Figure 1 (b)). Finally, the stability of the mean field analysis on limited data availability is demonstrated: Diffraction experiments under pressure or electric field yield a limited reciprocal space coverage. Here, we demonstrate the robustness of the proposed method against incomplete data sets.

(a)

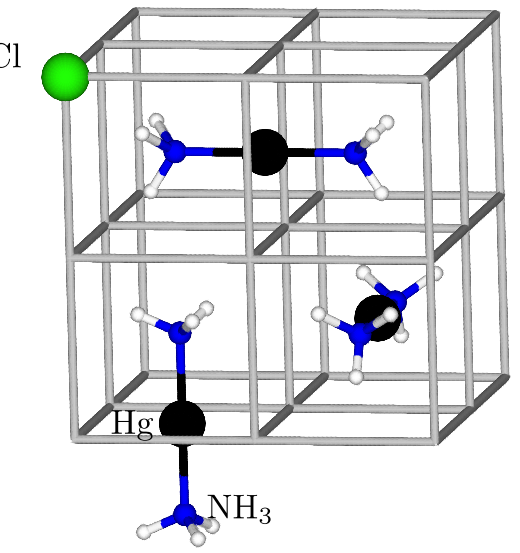

(b)

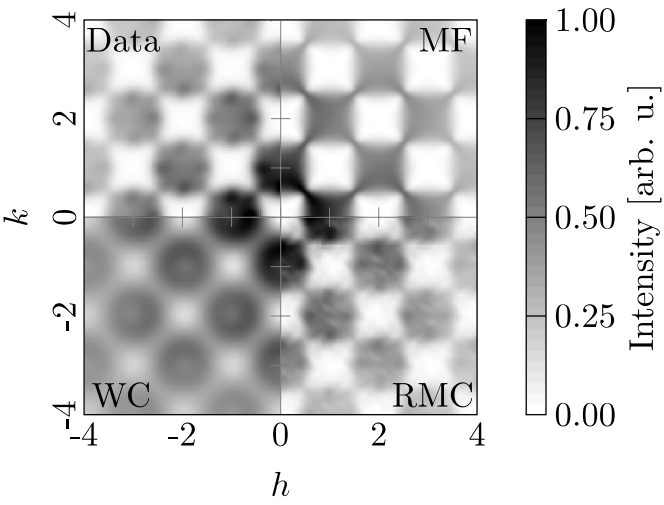

Figure 1. (a) $\mathrm{Hg}\left(\mathrm{NH}_{3}\right)_{2} \mathrm{Cl}_{2}$ [3], where the $\mathrm{Hg}$ is disordered over the cubic face centres. (b) Simulated data for a two dimensional analogue compared to refinements using mean field theory (MF), $\triangle \mathrm{PDF}$ analysis for the Warren-Cowley short-range order parameters

(WC) and reverse Monte Carlo modelling (RMC).

[1] Paddison, J.A.M., Stewart, J.R. et al. (2013). Phys. Rev. Letters. 110, 267207.

[2] Naya S. (1974) J. Phys. Soc. Jap. 37, 340-347.

[3] Lipscomb, W. N. (1953) Analytical Chemistry 25, 737-739.

Keywords: diffuse scattering; Mean field theory calculations 